MATHEMATICS OF COMPUTATION

Volume 68, Number 225, January 1999, Pages 291-305

S $0025-5718(99) 01000-5$

\title{
TAME AND WILD KERNELS OF QUADRATIC IMAGINARY NUMBER FIELDS
}

\author{
JERZY BROWKIN AND HERBERT GANGL
}

\begin{abstract}
For all quadratic imaginary number fields $F$ of discriminant $d>-5000$, we give the conjectural value of the order of Milnor's group (the tame kernel) $\mathrm{K}_{2} O_{F}$, where $O_{F}$ is the ring of integers of $F$. Assuming that the order is correct, we determine the structure of the group $\mathrm{K}_{2} \mathrm{O}_{F}$ and of its subgroup $W_{F}$ (the wild kernel). It turns out that the odd part of the tame kernel is cyclic (with one exception, $d=-3387$ ).
\end{abstract}

\section{INTRODUCTION}

Assuming Lichtenbaum's conjecture one can compute conjectural values of orders of the tame kernels $\mathrm{K}_{2} \mathrm{O}_{F}$ of quadratic imaginary number fields $F$.

Since in general these orders are not very large, and there are several results known concerning the $p$-rank of $K_{2} O_{F}$ and of its subgroup $W_{F}$ called the wild kernel, it is possible to determine the structure of these groups for the fields in question with discriminants $d>-5000$.

\section{Notations}

We use the following notation.

- $\quad F$ is a number field with $r_{1}$ real and $2 r_{2}$ complex embeddings.

- $\zeta_{F}(s)$ is the Dedekind zeta function of $F$.

- $O_{F}$ is the ring of integers of $F$.

- $K_{n} O_{F}$ is the $n$th Quillen $K$-group of $O_{F}$, and especially

- $K_{2} O_{F}$ is the Milnor group of $O_{F}$ (the tame kernel).

- $W_{F}$ is the Hilbert kernel of $F$ (the wild kernel).

- $e_{p}$ is the $p$-rank of $K_{2} O_{F}$, where $p$ is a prime or $p=4$.

- $w_{2}$ is the 2 -rank of $W_{F}$.

- $w(F)$ is the number of roots of unity in $F$.

- $\mathrm{Cl}(O)$ is the class group of a Dedekind ring $O$.

- $R_{m}(F)$ is a "twisted" version of the $m$ th Borel regulator (see [Bo1]), the "twisted" regulator map $r_{m}$ being a map

$$
r_{m}: K_{2 m-1} O_{F} \rightarrow\left[(2 \pi i)^{m-1} \mathbb{R}\right]^{d_{m}},
$$

Received by the editor January 3, 1997.

1991 Mathematics Subject Classification. Primary 11R11; Secondary 11R70, 11Y40, 19C99, $19 \mathrm{~F} 27$.

Key words and phrases. Tame kernel, wild kernel, quadratic imaginary fields, Lichtenbaum's conjecture.

The second author was supported by the Deutsche Forschungsgemeinschaft.

(C)1999 American Mathematical Society 
where $d_{m}=r_{2}$ for $m$ even, and $d_{m}=r_{1}+r_{2}$ for odd $m>1\left(d_{m}\right.$ is just the order of vanishing of $\zeta_{F}(s)$ at $s=1-m$ ). The image of $r_{m}$ is a lattice of covolume $R_{m}(F)$ - it differs from Borel's original one essentially by a power of $\pi$ ([Bo2]; there is also a shift $m \mapsto m+1$ compared to the original notation).

\section{Computing the value $\# K_{2} O_{F}$}

Borel proved that, up to a rational factor, $R_{m}(F)$ is equal to $\zeta_{F}^{*}(1-m)$, the first non-vanishing Taylor coefficient of $\zeta_{F}(s)$ at $s=1-m$. Lichtenbaum's conjecture [Li] (as modified by Borel [Bo1]) tries to interpret this rational factor and asks whether for all number fields and for any integer $m \geq 2$ there is a relation of the form

$$
\operatorname{res}_{s=1-m} \zeta_{F}(s)(s-1+m)^{-d_{m}(F)} \stackrel{?}{=} \pm \frac{\# K_{2 m-2}\left(O_{F}\right)}{\# K_{2 m-1}^{\text {ind }}\left(O_{F}\right)_{\text {tors }}} \cdot R_{m}(F)
$$

up to a power of 2 , where the subscript "tors" denotes the torsion part, "res" the residue, and "ind" the indecomposable part. $K_{2 m-2}\left(O_{F}\right)$ is known to be finite (Borel). There is some evidence for this conjecture, namely for $m=2$ and $F$ totally-real abelian it has been proved (up to a power of 2) by Mazur and Wiles $[\mathrm{M}-\mathrm{W}]$ as a consequence of their proof of the main conjecture of Iwasawa theory (in this case $R_{2}(F)=1$, though).

Recently Kolster, Nguyen Quang Do and Fleckinger ([KNF], Theorem 6.4) have proved a modified version of the conjecture (also up to a power of 2) for all abelian fields $F$ and $m \geq 2$. For imaginary quadratic fields $F$ and $m=2$, their result is equivalent to the above formula.

In what follows we assume $m=2$ and $F$ imaginary quadratic of discriminant $d$. In this case, the Lichtenbaum conjecture reads (using the functional equation for the zeta function and the fact that $\# K_{3}^{\text {ind }}\left(O_{F}\right)_{\text {tors }}$ is here always 24$)$

$$
\frac{3|d|^{3 / 2}}{\pi^{2} \cdot R_{2}(F)} \cdot \zeta_{F}(2) \stackrel{?}{=} \# K_{2}\left(O_{F}\right)
$$

up to a power of 2 .

Bloch [Bl] suggested and Suslin [Su] finally proved that Borel's regulator map can be given (at least rationally) in terms of the Bloch-Wigner dilogarithm $D_{2}(z)$ as a map on the Bloch group $\mathcal{B}(F)$; here $D_{2}(z)=\Im\left(L i_{2}(z)+\log |z| \log (1-z)\right)$, where $L i_{2}(z)=\sum_{n \geq 1} \frac{z^{n}}{n^{2}}$ is the classical dilogarithm function, defined for $|z|<1$ and analytically continued to $\mathbb{C}-[1, \infty)$, and $\mathcal{B}(F)$ is given in explicit form with generators and relations (cf. $[\mathrm{Su}])$ :

$$
\mathcal{B}(F)=\frac{\left\{\sum_{i} n_{i}\left[x_{i}\right] \mid \sum_{i} n_{i}\left(x_{i} \wedge\left(1-x_{i}\right)\right)=0 \in \bigwedge^{2} F^{\times}\right\}}{\left\langle[x]-[y]+\left[\frac{y}{x}\right]-\left[\frac{1-y}{1-x}\right]+\left[\frac{1-y^{-1}}{1-x^{-1}}\right] \mid x, y \in F^{\times}-\{1\}\right\rangle} .
$$

The dilogarithm $D_{2}(z)$ maps $\mathcal{B}(F)$ into a lattice in $\mathbb{R}$ whose covolume we denote by $D_{2}^{F}$. Thus, we can replace $R_{2}(F)$ in the formula above by $D_{2}^{F}$ and still hope for the following equality to hold (up to a universal factor):

$$
\frac{3|d|^{3 / 2}}{\pi^{2} \cdot D_{2}^{F}} \cdot \zeta_{F}(2) \stackrel{?}{=} \# K_{2}\left(O_{F}\right) \text {. }
$$

Note that in our formula we do not neglect powers of 2 .

The left hand side now can be computed numerically: we proceed by looking for elements $\xi \in \mathcal{B}(F)$ which are supported on exceptional $S$-units for some small 
set $S$ of primes in $F$, i.e. $\xi=\sum_{i} n_{i}\left[x_{i}\right]$ such that $\sum_{i} n_{i}\left(x_{i} \wedge\left(1-x_{i}\right)\right)=0$, and the principal ideals $\left(x_{i}\right)$ and $\left(1-x_{i}\right)$ are generated by $S$. The images $D_{2}(\xi)$ lie in a 1-dimensional lattice, therefore the numerically computed values should all be commensurable. The covolume $D_{2}^{F, S}$ of this lattice is an integral multiple of $D_{2}^{F}$ (to be precise, the covolume that we actually get depends not only on $S$ but also on the bounds that we impose on the valuations $v_{\mathcal{P}}\left(x_{i}\right)$ for $\mathcal{P} \in S$ in our search). If we have obtained hundreds of different values $D_{2}(\xi)$ there is a good chance that they already generate the correct lattice $D_{2}(\mathcal{B}(F))$ and give $D_{2}^{F}$ exactly.

Our program, written in PARI [BBCO], performs the above calculations successively for an increasing set of primes and stops if the corresponding $D_{2}^{F, S}$ stabilizes, i.e. if the same covolume occurs for $S$ and $S^{\prime} \supsetneqq S$.

The orders in the case of small discriminants have been determined by Tate [Ta] (for $|d| \leq 15)$, Skałba [Sk] $(d=-19,-20)$, and Qin [Q2], [Q3] $(d=-24,-35)$, and they coincide with ours. Furthermore, the entries of a former (shorter) table [Ga] were not only compatible with the structural theoretical results known at the time but even suggested several conjectures, most of which have been proved in the meantime ([B-92], [C-H], [Q1]).

Our approach is very similar to that of Grayson $[\mathrm{Gr}]$, only we don't have to restrict ourselves to class number one, and our program works even for quite large discriminants (e.g., for $F=\mathbb{Q}(\sqrt{-2000004})$ we obtain $\left.\# K_{2} O_{F}=4\right)$.

The program is freely available from the second author via e-mail, together with some remarks on the modification of the parameters.

\section{Determining the structure}

In order to establish the actual structure of the tame and wild kernel we apply the following results: let $d^{\prime}=d / \operatorname{gcd}(4, d)$.

(1) The index $i_{F}:=\left(K_{2} O_{F}: W_{F}\right)$ always divides 6 . More precisely,

$$
\begin{aligned}
& 2 \mid i_{F} \quad \text { iff } \quad d^{\prime} \equiv \pm 1(\bmod 8), \\
& 3 \mid i_{F} \quad \text { iff } \quad d \equiv-3(\bmod 9), \quad d \neq-3 \text {. }
\end{aligned}
$$

(See [B-82], Table 1.)

(2) The 2-rank of the tame and wild kernels can be computed easily:

$$
e_{2}= \begin{cases}t, & \text { if every odd prime divisor of } d \text { is } \equiv \pm 1 \quad(\bmod 8), \\ t-1, & \text { otherwise, }\end{cases}
$$

where $t$ is the number of odd prime divisors of $d$; and

$$
w_{2}= \begin{cases}e_{2}, & \text { if } d^{\prime} \not \equiv 1 \quad(\bmod 8), \\ e_{2}-1, & \text { otherwise }\end{cases}
$$

(See [B-S], Theorem 4.)

(3) The 4-rank of the tame kernel can be easily determined using the results of [Q1], at least if the number of odd prime divisors of $d$ does not exceed 3 .

The $p$-rank of $K_{2} O_{F}$, for odd $p$, is related to the $p$-rank of the class group of an appropriate number field as follows.

(4) Let $E_{3}=\mathbb{Q}(\sqrt{-3 d})$ and $e_{3}^{\prime}=3$-rank $C l\left(O_{E_{3}}\right)$. Then

$$
e_{3}=e_{3}^{\prime}, \quad \text { if } d \not \equiv-3 \quad(\bmod 9),
$$




$$
\max \left(1, e_{3}^{\prime}\right) \leq e_{3} \leq e_{3}^{\prime}+1, \quad \text { otherwise. }
$$

(See [B-92], Theorem 5.6.)

(5) Let $E_{5}=\mathbb{Q}(\sqrt{5 d})$, and $e_{5}^{\prime}=5$-rank $C l\left(O_{E_{5}}\right)$. Then $e_{5} \leq e_{5}^{\prime}$. (See [B-92], Theorem 5.4.)

(6) For $p>5$, where $p$ is a regular prime, let $E_{p}$ be the maximal real subfield of the field $F\left(\zeta_{p}\right)$, and let $e_{p}^{\prime}=p$-rank $C l\left(O_{E_{p}}\right)$. Then $e_{p} \leq e_{p}^{\prime}$. (See [B-92], Theorem 5.4.)

\section{EXAMPLES}

As above, let $d^{\prime}=d / \operatorname{gcd}(4, d)$.

1) For $d=-644$, we have $\# K_{2} O_{F}=32$ (conjecturally), and $e_{2}=2, w_{2}=2$. Moreover $e_{4}=1$, since $644=4 \cdot 7 \cdot 23$, and $7 \equiv 23 \equiv 7(\bmod 8)$, see [Q1]. Finally, $\left(K_{2} O_{F}: W_{F}\right)=2$, since $d^{\prime}=-161 \equiv 7(\bmod 8)$ and $d \not \equiv-3(\bmod 9)$. It follows that

$$
K_{2} O_{F}=\mathbb{Z} / 2 \times \mathbb{Z} / 16 \text { and } W_{F}=\mathbb{Z} / 2 \times \mathbb{Z} / 8
$$

2) For $d=-255$ we have $\# K_{2} O_{F}=12$ (conjecturally). Moreover $e_{2}=2, w_{2}=1$, and $d \equiv-3(\bmod 9)$. Therefore

$$
K_{2} O_{F}=\mathbb{Z} / 2 \times \mathbb{Z} / 2 \times \mathbb{Z} / 3 \text { and } W_{F}=\mathbb{Z} / 2 .
$$

3) For $d=-759$, we have $\# K_{2} O_{F}=36$ (conjecturally), and $e_{2}=2, w_{2}=1$, and $d \equiv-3(\bmod 9)$. Moreover, for

$$
E_{3}=\mathbb{Q}(\sqrt{3 d})=\mathbb{Q}(\sqrt{-253}),
$$

we have 3-rank $C l\left(O_{E_{3}}\right)=0$. Therefore

$$
K_{2} O_{F}=\mathbb{Z} / 2 \times \mathbb{Z} / 2 \times \mathbb{Z} / 9 \text { and } W_{F}=\mathbb{Z} / 2 \times \mathbb{Z} / 3 .
$$

4) For $d=-2395$, we have $\# K_{2} O_{F}=25$ (conjecturally). Moreover, for $E_{5}=$ $\mathbb{Q}(\sqrt{5 d})=\mathbb{Q}(\sqrt{-479})$, we have 5 -rank $C l\left(O_{E_{5}}\right)=1$. Therefore, using (5),

$$
K_{2} O_{F}=W_{F}=\mathbb{Z} / 25 \text {. }
$$

5) For $d=-1832$, we have $\# K_{2} O_{F}=49$ (conjecturally). The maximal real subfield $E_{7}$ of the field $F\left(\zeta_{7}\right)=\mathbb{Q}\left(\sqrt{-d}, \zeta_{7}\right)$ is generated over $\mathbb{Q}$ by a root of the polynomial

$$
f(x)=x^{6}+7 d x^{4}+14 d^{2} x^{2}+7 d^{3} .
$$

In our case

$$
e_{7}^{\prime}=7-\operatorname{rank} C l\left(O_{E_{7}}\right)=1 .
$$

Therefore, in view of (6),

$$
K_{2} O_{F}=W_{F}=\mathbb{Z} / 49 .
$$

\section{Description of the table}

In the first column there is the negative discriminant $d$. The last two columns give the structure of the tame and the wild kernel of the corresponding field. In these columns a single number $n$ denotes the cyclic group of order $n$, and a sequence $\left(n_{1}, n_{2}, \ldots\right)$ denotes the direct sum of cyclic groups of orders $n_{1}, n_{2}, \ldots$.

The last two columns contain correct results provided the conjectural value of $\# K_{2} O_{F}$ is correct. 
TABLE 1. Table of tame and wild kernels for imaginary quadratic number fields of discriminant $d>-5000$ (conjectural values)

\begin{tabular}{|c|c|c|}
\hline$d$ & tame & wild \\
\hline-3 & 1 & 1 \\
-4 & 1 & 1 \\
-7 & 2 & 1 \\
-8 & 1 & 1 \\
-11 & 1 & 1 \\
-15 & 2 & 1 \\
-19 & 1 & 1 \\
-20 & 1 & 1 \\
-23 & 2 & 1 \\
-24 & 1 & 1 \\
-31 & 2 & 1 \\
-35 & 2 & 2 \\
-39 & $(2,3)$ & 1 \\
-40 & 1 & 1 \\
-43 & 1 & 1 \\
-47 & 2 & 1 \\
-51 & 2 & 2 \\
-52 & 1 & 1 \\
-55 & 2 & 1 \\
-56 & 2 & 2 \\
-59 & 1 & 1 \\
-67 & 1 & 1 \\
-68 & 8 & 4 \\
-71 & 2 & 1 \\
-79 & 2 & 1 \\
-83 & 1 & 1 \\
-84 & $(2,3)$ & 2 \\
-87 & 2 & 1 \\
-88 & 1 & 1 \\
-91 & 2 & 2 \\
-95 & 2 & 1 \\
-103 & 2 & 1 \\
-104 & 1 & 1 \\
-107 & 3 & 3 \\
-111 & $(2,3)$ & 1 \\
-115 & 2 & 2 \\
-116 & 1 & 1 \\
-119 & $(2,2)$ & 2 \\
-120 & $(2,3)$ & 2 \\
-123 & 2 & 2 \\
-127 & 2 & 1 \\
-131 & 1 & 1 \\
-132 & 4 & 2 \\
-136 & 4 & 4 \\
-139 & 1 & 1 \\
-143 & 2 & 1 \\
-148 & 1 & 1 \\
-151 & 2 & 1 \\
-152 & 1 & 1 \\
-155 & 2 & 2 \\
-159 & 2 & 1 \\
\hline & & \\
\hline
\end{tabular}

\begin{tabular}{|c|c|c|}
\hline$d$ & tame & wild \\
\hline-163 & 1 & 1 \\
-164 & 4 & 2 \\
-167 & 2 & 1 \\
-168 & 2 & 2 \\
-179 & 1 & 1 \\
-183 & $(2,3)$ & 1 \\
-184 & 2 & 2 \\
-187 & 2 & 2 \\
-191 & 2 & 1 \\
-195 & $(2,2)$ & $(2,2)$ \\
-199 & 2 & 1 \\
-203 & 2 & 2 \\
-211 & 1 & 1 \\
-212 & 1 & 1 \\
-215 & 2 & 1 \\
-219 & $(4,3)$ & 4 \\
-223 & 2 & 1 \\
-227 & 1 & 1 \\
-228 & $(4,3)$ & 2 \\
-231 & $(2,2)$ & 2 \\
-232 & 1 & 1 \\
-235 & 2 & 2 \\
-239 & 2 & 1 \\
-244 & 1 & 1 \\
-247 & 2 & 1 \\
-248 & 2 & 2 \\
-251 & 1 & 1 \\
-255 & $(2,2,3)$ & 2 \\
-259 & 2 & 2 \\
-260 & 4 & 2 \\
-263 & 2 & 1 \\
-264 & $(2,3)$ & 2 \\
-267 & 2 & 2 \\
-271 & 2 & 1 \\
-276 & 2 & 2 \\
-280 & 2 & 2 \\
-283 & 1 & 1 \\
-287 & $(2,2)$ & 2 \\
-291 & $(4,3)$ & 4 \\
-292 & 4 & 2 \\
-295 & 2 & 1 \\
-296 & 1 & 1 \\
-299 & 2 & 2 \\
-303 & $(2,11)$ & 11 \\
-307 & 1 & 1 \\
-308 & 2 & 2 \\
-311 & 2 & 1 \\
-312 & 2 & 2 \\
-319 & 2 & 1 \\
-323 & 4 & 4 \\
-327 & $(2,3)$ & 1 \\
\hline & &
\end{tabular}

\begin{tabular}{|c|c|c|}
\hline$d$ & tame & wild \\
\hline-328 & 2 & 2 \\
-331 & 3 & 3 \\
-335 & 2 & 1 \\
-339 & 2 & 2 \\
-340 & 2 & 2 \\
-344 & 1 & 1 \\
-347 & 1 & 1 \\
-355 & 2 & 2 \\
-356 & 4 & 2 \\
-359 & 2 & 1 \\
-367 & $(2,3)$ & 3 \\
-371 & 2 & 2 \\
-372 & $(2,3)$ & 2 \\
-376 & 2 & 2 \\
-379 & 1 & 1 \\
-383 & 2 & 1 \\
-388 & 8 & 4 \\
-391 & $(2,2)$ & 2 \\
-395 & 2 & 2 \\
-399 & $(2,4,3)$ & 4 \\
-403 & 2 & 2 \\
-404 & 1 & 1 \\
-407 & 2 & 1 \\
-408 & $(2,3)$ & 2 \\
-411 & 2 & 2 \\
-415 & 2 & 1 \\
-419 & 3 & 3 \\
-420 & $(2,4)$ & $(2,2)$ \\
-424 & 1 & 1 \\
-427 & 2 & 2 \\
-431 & 2 & 1 \\
-435 & $(2,2,3)$ & $(2,2)$ \\
-436 & 1 & 1 \\
-439 & 2 & 1 \\
-440 & 2 & 2 \\
-443 & 1 & 1 \\
-447 & 2 & 1 \\
-451 & 2 & 2 \\
-452 & 8 & 4 \\
-455 & $(2,2)$ & 2 \\
-456 & 2 & 2 \\
-463 & 2 & 1 \\
-467 & 1 & 1 \\
-471 & $(2,3)$ & 1 \\
-472 & 5 & 5 \\
-479 & $(2,7)$ & 7 \\
-483 & $(2,2)$ & $(2,2)$ \\
-487 & 2 & 1 \\
-488 & 1 & 1 \\
-491 & 13 & 13 \\
-499 & 1 & 1 \\
\hline & &
\end{tabular}


Table 1. (Continued)

\begin{tabular}{|c|c|c|c|c|c|c|c|c|}
\hline$d$ & tame & wild & $d$ & tame & wild & $d$ & tame & wild \\
\hline-503 & $(2,3)$ & 3 & -667 & 2 & 2 & -831 & $(2,3)$ & 1 \\
\hline-511 & $(2,2)$ & 2 & -671 & 2 & 1 & -835 & $(2,3)$ & $(2,3)$ \\
\hline-515 & 2 & 2 & -679 & $(2,2,5)$ & $(2,5)$ & -836 & 4 & 2 \\
\hline-516 & $(4,3)$ & 2 & -680 & 2 & 2 & -839 & 2 & 1 \\
\hline-519 & 2 & 1 & -683 & 1 & 1 & -840 & $(2,2,3)$ & $(2,2)$ \\
\hline-520 & 2 & 2 & -687 & $(2,3)$ & 1 & -843 & 2 & 2 \\
\hline-523 & 1 & 1 & -691 & 1 & 1 & -851 & 2 & 2 \\
\hline-527 & $(2,2)$ & 2 & -692 & 1 & 1 & -852 & 2 & 2 \\
\hline-532 & 2 & 2 & -695 & 2 & 1 & -856 & 1 & 1 \\
\hline-535 & 2 & 1 & -696 & $(2,3,7)$ & $(2,7)$ & -859 & 1 & 1 \\
\hline-536 & 1 & 1 & -699 & 2 & 2 & -863 & $(2,3)$ & 3 \\
\hline-543 & $(2,3)$ & 1 & -703 & $(2,37)$ & 37 & -868 & $(2,4)$ & $(2,2)$ \\
\hline-547 & 1 & 1 & -707 & 2 & 2 & -871 & 2 & 1 \\
\hline-548 & 4 & 2 & -708 & 4 & 2 & -872 & 1 & 1 \\
\hline-551 & 2 & 1 & -712 & 2 & 2 & -879 & $(2,5)$ & 5 \\
\hline-552 & $(2,3)$ & 2 & -715 & $(2,2)$ & $(2,2)$ & -883 & 1 & 1 \\
\hline-555 & $(2,2,7)$ & $(2,2,7)$ & -719 & 2 & 1 & -884 & 4 & 4 \\
\hline-559 & 2 & 1 & -723 & $(4,3)$ & 4 & -887 & $(2,5)$ & 5 \\
\hline-563 & 1 & 1 & -724 & 1 & 1 & -888 & 2 & 2 \\
\hline-564 & 2 & 2 & -727 & 2 & 1 & -895 & 2 & 1 \\
\hline-568 & 2 & 2 & -728 & 2 & 2 & -899 & 2 & 2 \\
\hline-571 & 5 & 5 & -731 & 4 & 4 & -903 & $(2,2,3)$ & 2 \\
\hline-579 & $(4,3)$ & 4 & -739 & 1 & 1 & -904 & 4 & 4 \\
\hline-580 & 4 & 2 & -740 & 4 & 2 & -907 & 1 & 1 \\
\hline-583 & $(2,17)$ & 17 & -743 & 2 & 1 & -911 & 2 & 1 \\
\hline-584 & 2 & 2 & -744 & 2 & 2 & -915 & $(2,2)$ & $(2,2)$ \\
\hline-587 & 1 & 1 & -751 & 2 & 1 & -916 & 1 & 1 \\
\hline-591 & 2 & 1 & -755 & $(2,41)$ & $(2,41)$ & -919 & 2 & 1 \\
\hline-595 & $(2,2)$ & $(2,2)$ & -759 & $(2,2,9)$ & $(2,3)$ & -920 & 2 & 2 \\
\hline-596 & 1 & 1 & -760 & 2 & 2 & -923 & 2 & 2 \\
\hline-599 & 2 & 1 & -763 & 2 & 2 & -932 & $(4,5)$ & $(2,5)$ \\
\hline-607 & 2 & 1 & -767 & 2 & 1 & -935 & $(2,2)$ & 2 \\
\hline-611 & 2 & 2 & -771 & $(2,3)$ & $(2,3)$ & -939 & $(4,3)$ & 4 \\
\hline-615 & $(2,2,3)$ & 2 & -772 & 8 & 4 & -943 & $(2,2)$ & 2 \\
\hline-616 & 2 & 2 & -776 & 4 & 4 & -947 & 1 & 1 \\
\hline-619 & 1 & 1 & -779 & 2 & 2 & -948 & $(2,3)$ & 2 \\
\hline-623 & $(2,2)$ & 2 & -787 & 1 & 1 & -951 & 2 & 1 \\
\hline-627 & $(2,2)$ & $(2,2)$ & -788 & 1 & 1 & -952 & $(2,2)$ & $(2,2)$ \\
\hline-628 & 1 & 1 & -791 & $(2,2)$ & 2 & -955 & 2 & 2 \\
\hline-631 & 2 & 1 & -795 & $(2,2,3)$ & $(2,2)$ & -959 & $(2,4)$ & 4 \\
\hline-632 & 2 & 2 & -799 & $(2,4)$ & 4 & -964 & 8 & 4 \\
\hline-635 & 2 & 2 & -803 & 2 & 2 & -967 & 2 & 1 \\
\hline-643 & 3 & 3 & -804 & $(4,9)$ & $(2,3)$ & -971 & 5 & 5 \\
\hline-644 & $(2,16)$ & $(2,8)$ & -807 & 2 & 1 & -979 & 4 & 4 \\
\hline-647 & 2 & 1 & -808 & 1 & 1 & -983 & 2 & 1 \\
\hline-651 & $(2,2,3)$ & $(2,2)$ & -811 & 1 & 1 & -984 & $(2,3)$ & 2 \\
\hline-655 & 2 & 1 & -815 & 2 & 1 & -987 & $(2,2)$ & $(2,2)$ \\
\hline-659 & 1 & 1 & -820 & 4 & 4 & -991 & 2 & 1 \\
\hline-660 & $(2,2,3)$ & $(2,2)$ & -823 & 2 & 1 & -995 & 2 & 2 \\
\hline-663 & $(2,2)$ & 2 & -824 & 2 & 2 & -996 & 4 & 2 \\
\hline-664 & 1 & 1 & -827 & 1 & 1 & -1003 & 4 & 4 \\
\hline
\end{tabular}


Table 1. (Continued)

\begin{tabular}{|c|c|c|c|c|c|c|c|c|}
\hline$d$ & tame & wild & $d$ & tame & wild & $d$ & tame & wild \\
\hline \begin{tabular}{l|l|}
-1007 \\
\end{tabular} & $(2,3)$ & 3 & -1171 & 1 & 1 & \begin{tabular}{|l|}
-1343 \\
\end{tabular} & $(2,2)$ & 2 \\
\hline-1011 & $(4,3)$ & 4 & -1172 & 1 & 1 & -1347 & 2 & 2 \\
\hline-1012 & 2 & 2 & -1187 & 7 & 7 & -1348 & 16 & 8 \\
\hline-1015 & $(2,2)$ & 2 & $|-1191|$ & $(2,27)$ & 9 & -1351 & $(2,4)$ & 4 \\
\hline-1016 & $(2,13)$ & $(2,13)$ & -1192 & 3 & 3 & -1355 & $(2,3)$ & $(2,3)$ \\
\hline-1019 & 1 & 1 & $-1195 \mid$ & 2 & 2 & -1363 & 2 & 2 \\
\hline-1023 & $(2,16)$ & 16 & $\mid-1199$ & 2 & 1 & -1364 & 2 & 2 \\
\hline-1027 & 2 & 2 & -1203 & 2 & 2 & -1367 & 2 & 1 \\
\hline-1028 & 8 & 4 & -1204 & 2 & 2 & -1371 & $(4,3,5)$ & $(4,5)$ \\
\hline-1031 & 2 & 1 & $\mid-1207$ & $(2,2)$ & 2 & -1379 & 2 & 2 \\
\hline-1032 & 2 & 2 & -1208 & $(2,3)$ & $(2,3)$ & -1380 & $(2,4,3)$ & $(2,2)$ \\
\hline-1039 & 2 & 1 & $|-1211|$ & 2 & 2 & $\mid-1383$ & 2 & 1 \\
\hline-1043 & 2 & 2 & $|-1219|$ & 2 & 2 & -1384 & 1 & 1 \\
\hline-1047 & $(2,3)$ & 1 & -1220 & 4 & 2 & $|-1387|$ & $(4,11)$ & $(4,11)$ \\
\hline-1048 & $(3,11)$ & $(3,11)$ & -1223 & 2 & 1 & -1391 & 2 & 1 \\
\hline-1051 & 1 & 1 & -1227 & $(4,3)$ & 4 & -1396 & 1 & 1 \\
\hline-1055 & 2 & 1 & -1231 & 2 & 1 & -1399 & 2 & 1 \\
\hline-1059 & 2 & 2 & -1235 & $(2,2,11)$ & $(2,2,11)$ & -1403 & 2 & 2 \\
\hline-1060 & 4 & 2 & -1236 & $(2,9)$ & $(2,3)$ & -1407 & $(2,2,3)$ & 2 \\
\hline-1063 & $(2,29)$ & 29 & -1239 & $(2,8)$ & 8 & -1411 & 4 & 4 \\
\hline-1064 & 2 & 2 & $|-1240|$ & $(2,17)$ & $(2,17)$ & $\mid-1412$ & 16 & 8 \\
\hline-1067 & 4 & 4 & -1243 & $(4,7)$ & $(4,7)$ & -1415 & 2 & 1 \\
\hline-1076 & 1 & 1 & -1247 & 2 & 1 & -1416 & $(2,3)$ & 2 \\
\hline-1079 & 2 & 1 & $|-1252|$ & 4 & 2 & -1419 & $(2,2,9)$ & $(2,2,9)$ \\
\hline-1087 & $(2,3)$ & 3 & $\mid-1255$ & 2 & 1 & -1423 & 2 & 1 \\
\hline-1091 & 1 & 1 & -1256 & 5 & 5 & -1427 & 3 & 3 \\
\hline-1092 & $(2,4,3)$ & $(2,2)$ & -1259 & 1 & 1 & -1428 & $(2,2)$ & $(2,2)$ \\
\hline-1095 & $(2,2)$ & 2 & $\mid-1263$ & $(2,3)$ & 1 & $\mid-1432$ & 1 & 1 \\
\hline-1096 & $(2,31)$ & $(2,31)$ & $-1267 \mid$ & 2 & 2 & -1435 & $(2,2)$ & $(2,2)$ \\
\hline-1099 & 2 & 2 & -1268 & 1 & 1 & -1439 & 2 & 1 \\
\hline-1103 & $(2,5)$ & 5 & -1271 & $(2,2)$ & 2 & -1443 & $(2,4,3)$ & $(2,4)$ \\
\hline-1108 & 1 & 1 & $\mid-1272$ & $(2,9)$ & $(2,3)$ & -1447 & 2 & 1 \\
\hline-1111 & 2 & 1 & -1279 & 2 & 1 & -1448 & 3 & 3 \\
\hline-1112 & 5 & 5 & -1283 & 5 & 5 & -1451 & 1 & 1 \\
\hline-1115 & 2 & 2 & $-1284 \mid$ & 4 & 2 & -1455 & $(2,2)$ & 2 \\
\hline-1119 & $(2,3)$ & 1 & $\mid-1288$ & $(2,4)$ & $(2,4)$ & -1459 & 1 & 1 \\
\hline-1123 & 1 & 1 & -1291 & 3 & 3 & -1460 & 2 & 2 \\
\hline-1124 & 4 & 2 & $|-1295|$ & $(2,2)$ & 2 & -1463 & $(2,2)$ & 2 \\
\hline-1128 & $(2,3)$ & 2 & $\mid-1299$ & $(8,3)$ & 8 & -1464 & 2 & 2 \\
\hline-1131 & $(2,2)$ & $(2,2)$ & -1303 & 2 & 1 & -1471 & $(2,7)$ & 7 \\
\hline-1135 & $(2,7)$ & 7 & $-1304 \mid$ & 1 & 1 & -1479 & $(2,2,3)$ & 2 \\
\hline-1139 & 4 & 4 & -1307 & 1 & 1 & -1480 & 2 & 2 \\
\hline-1140 & $(2,2)$ & $(2,2)$ & $|-1311|$ & $(2,2)$ & 2 & -1483 & 1 & 1 \\
\hline-1144 & 2 & 2 & $\mid-1315$ & 2 & 2 & -1487 & $(2,5)$ & 5 \\
\hline-1147 & 2 & 2 & -1316 & $(2,4)$ & $(2,2)$ & -1491 & $(2,2)$ & $(2,2)$ \\
\hline-1151 & 2 & 1 & $\mid-1319$ & $(2,3)$ & 3 & $\mid-1492$ & 1 & 1 \\
\hline-1155 & $(2,2,2,3)$ & $(2,2,2)$ & $-1320 \mid$ & $(2,2,13)$ & $(2,2,13)$ & -1495 & $(2,2,17)$ & $(2,17)$ \\
\hline-1159 & 2 & 1 & -1327 & $(2,3)$ & 3 & -1496 & 2 & 2 \\
\hline-1160 & 2 & 2 & $\mid-1335$ & $(2,2,3)$ & 2 & -1499 & 1 & 1 \\
\hline-1163 & 1 & 1 & -1336 & 2 & 2 & -1507 & 4 & 4 \\
\hline-1167 & 2 & 1 & -1339 & 2 & 2 & -1508 & 4 & 2 \\
\hline
\end{tabular}


Table 1. (Continued)

\begin{tabular}{|c|c|c|c|c|c|c|c|c|}
\hline$d$ & tame & wild & $d$ & tame & wild & $d$ & tame & wild \\
\hline $\mid-1511$ & 2 & 1 & $\mid-1671$ & 2 & 1 & \begin{tabular}{|l|}
-1839 \\
\end{tabular} & $(2,3,5)$ & 5 \\
\hline $\mid-1515$ & $(2,2,9)$ & $(2,2,3)$ & $\mid-1672$ & 2 & 2 & -1843 & $(2,3)$ & $(2,3)$ \\
\hline-1523 & 7 & 7 & $\mid-1679$ & $(2,4)$ & 4 & -1844 & 1 & 1 \\
\hline-1524 & $(2,3)$ & 2 & -1684 & 1 & 1 & -1847 & $(2,23)$ & 23 \\
\hline-1527 & 2 & 1 & $|-1687|$ & $(2,2)$ & 2 & -1848 & $(2,2,3)$ & $(2,2)$ \\
\hline-1528 & 2 & 2 & $\mid-1688$ & 1 & 1 & -1851 & 2 & 2 \\
\hline-1531 & 1 & 1 & $\mid-1691$ & $(2,3)$ & $(2,3)$ & -1855 & $(2,2)$ & 2 \\
\hline-1535 & 2 & 1 & $\mid-1695$ & $(2,2,3)$ & 2 & -1860 & $(2,4)$ & $(2,2)$ \\
\hline-1540 & $(2,4)$ & $(2,2)$ & $\mid-1699$ & 1 & 1 & -1864 & 2 & 2 \\
\hline-1543 & 2 & 1 & -1703 & 2 & 1 & -1867 & 1 & 1 \\
\hline-1544 & 4 & 4 & -1704 & $(2,3)$ & 2 & -1871 & $(2,3)$ & 3 \\
\hline-1547 & $(2,2,3)$ & $(2,2,3)$ & -1707 & 2 & 2 & -1876 & 2 & 2 \\
\hline-1551 & $(2,2,3)$ & 2 & -1711 & 2 & 1 & -1879 & $(2,3)$ & 3 \\
\hline-1555 & 2 & 2 & -1716 & $(2,2)$ & $(2,2)$ & -1880 & 2 & 2 \\
\hline-1556 & 1 & 1 & $|-1720|$ & 2 & 2 & -1883 & 2 & 2 \\
\hline-1559 & 2 & 1 & -1723 & 7 & 7 & -1887 & $(2,2)$ & 2 \\
\hline-1560 & $(2,2,3)$ & $(2,2)$ & -1727 & 2 & 1 & -1891 & 2 & 2 \\
\hline-1563 & 2 & 2 & -1731 & $(4,3)$ & 4 & -1892 & 4 & 2 \\
\hline $\mid-1567$ & 2 & 1 & $\mid-1732$ & 8 & 4 & -1895 & $(2,3)$ & 3 \\
\hline-1571 & 7 & 7 & -1735 & $(2,5)$ & 5 & -1896 & 2 & 2 \\
\hline $\mid-1572$ & $(4,5)$ & $(2,5)$ & -1736 & $(2,2,7)$ & $(2,2,7)$ & -1903 & 2 & 1 \\
\hline-1576 & 1 & 1 & -1739 & 2 & 2 & -1907 & 1 & 1 \\
\hline $\mid-1579$ & 1 & 1 & -1743 & $(2,4)$ & 4 & -1912 & 2 & 2 \\
\hline-1583 & $(2,27)$ & 27 & -1747 & 1 & 1 & -1915 & 2 & 2 \\
\hline-1588 & 3 & 3 & -1748 & 2 & 2 & -1919 & 2 & 1 \\
\hline-1591 & 2 & 1 & -1751 & $(2,2)$ & 2 & -1923 & 2 & 2 \\
\hline-1592 & 2 & 2 & -1752 & 4 & 4 & -1924 & 4 & 2 \\
\hline-1595 & $(2,2)$ & $(2,2)$ & -1759 & 2 & 1 & -1927 & $(2,2)$ & 2 \\
\hline-1599 & $(2,2)$ & 2 & $\mid-1763$ & 4 & 4 & -1928 & 4 & 4 \\
\hline-1603 & 2 & 2 & $-1767 \mid$ & $(2,2,3)$ & 2 & -1931 & 1 & 1 \\
\hline-1604 & 8 & 4 & -1768 & 4 & 4 & -1939 & 2 & 2 \\
\hline-1607 & 2 & 1 & -1771 & $(2,2)$ & $(2,2)$ & -1940 & 2 & 2 \\
\hline-1608 & 2 & 2 & -1779 & 2 & 2 & -1943 & 2 & 1 \\
\hline-1615 & $(2,2)$ & 2 & -1780 & 4 & 4 & -1947 & $(2,4,3)$ & $(2,4)$ \\
\hline-1619 & 3 & 3 & -1783 & 2 & 1 & -1951 & $(2,3,5)$ & $(3,5)$ \\
\hline $\mid-1623$ & $(2,3)$ & 1 & -1784 & 2 & 2 & -1955 & $(2,2)$ & $(2,2)$ \\
\hline-1624 & 2 & 2 & -1787 & 1 & 1 & -1956 & $(4,3)$ & 2 \\
\hline-1627 & 1 & 1 & -1795 & 2 & 2 & -1959 & 2 & 1 \\
\hline-1631 & $(2,2)$ & 2 & -1796 & $(8,7)$ & $(4,7)$ & -1963 & 2 & 2 \\
\hline-1635 & $(2,2)$ & $(2,2)$ & -1799 & $(2,2)$ & 2 & -1967 & $(2,2,3)$ & $(2,3)$ \\
\hline-1636 & $(4,19)$ & $(2,19)$ & -1803 & $(4,3,13)$ & $(4,13)$ & -1972 & 2 & 2 \\
\hline-1639 & 2 & 1 & $\mid-1807$ & 2 & 1 & -1976 & 2 & 2 \\
\hline-1640 & 4 & 4 & $\mid-1811$ & 1 & 1 & -1979 & 1 & 1 \\
\hline $\mid-1643$ & 2 & 2 & $\mid-1812$ & $(2,3)$ & 2 & -1983 & $(2,3)$ & 1 \\
\hline-1651 & 2 & 2 & $\mid-1816$ & 1 & 1 & -1987 & 1 & 1 \\
\hline-1652 & 2 & 2 & $\mid-1819$ & 2 & 2 & -1988 & $(2,8)$ & $(2,4)$ \\
\hline-1655 & 2 & 1 & -1823 & 2 & 1 & -1991 & 2 & 1 \\
\hline-1659 & $(2,2,3)$ & $(2,2)$ & -1828 & 4 & 2 & -1992 & $(2,3)$ & 2 \\
\hline-1663 & 2 & 1 & $\mid-1831$ & 2 & 1 & -1995 & $(2,2,2)$ & $(2,2,2)$ \\
\hline-1667 & 83 & 83 & -1832 & 49 & 49 & -1999 & 2 & 1 \\
\hline-1668 & $(4,9)$ & $(2,3)$ & -1835 & 2 & 2 & -2003 & 1 & 1 \\
\hline
\end{tabular}


Table 1. (Continued)

\begin{tabular}{|c|c|c|c|c|c|c|c|c|}
\hline$d$ & tame & wild & $d$ & tame & wild & $d$ & tame & wild \\
\hline-2004 & 2 & 2 & -2171 & 2 & 2 & -2344 & 3 & 3 \\
\hline-2008 & 1 & 1 & -2179 & 25 & 25 & -2347 & 1 & 1 \\
\hline-2011 & 1 & 1 & -2180 & 4 & 2 & -2351 & $(2,3)$ & 3 \\
\hline-2015 & $(2,2)$ & 2 & -2183 & $(2,3)$ & 3 & -2355 & $(2,2,9)$ & $(2,2,9)$ \\
\hline-2019 & $(16,3)$ & 16 & -2184 & $(2,2)$ & $(2,2)$ & -2356 & 2 & 2 \\
\hline-2020 & 4 & 2 & -2191 & $(2,2)$ & 2 & -2359 & $(2,2)$ & 2 \\
\hline-2024 & $(2,7)$ & $(2,7)$ & -2195 & $(2,5)$ & $(2,5)$ & -2360 & 2 & 2 \\
\hline-2027 & 1 & 1 & -2199 & $(2,3)$ & 1 & -2363 & 2 & 2 \\
\hline-2031 & 2 & 1 & -2203 & 1 & 1 & -2371 & 1 & 1 \\
\hline-2035 & $(2,4)$ & $(2,4)$ & -2207 & 2 & 1 & -2372 & 16 & 8 \\
\hline-2036 & 3 & 3 & -2211 & $(2,8)$ & $(2,8)$ & -2379 & $(2,4,3)$ & $(2,4)$ \\
\hline-2039 & 2 & 1 & -2212 & $(2,4)$ & $(2,2)$ & -2383 & 2 & 1 \\
\hline-2040 & $(2,2)$ & $(2,2)$ & -2215 & $(2,5,23)$ & $(5,23)$ & -2387 & $(2,2)$ & $(2,2)$ \\
\hline-2047 & $(2,2)$ & 2 & -2216 & 1 & 1 & -2388 & $(2,3)$ & 2 \\
\hline-2051 & $(2,3)$ & $(2,3)$ & -2219 & 2 & 2 & -2391 & 2 & 1 \\
\hline-2055 & $(2,2,3)$ & 2 & -2227 & $(2,3)$ & $(2,3)$ & -2392 & $(2,7)$ & $(2,7)$ \\
\hline-2056 & 4 & 4 & -2228 & 1 & 1 & -2395 & $(2,25)$ & $(2,25)$ \\
\hline-2059 & 2 & 2 & -2231 & $(2,2)$ & 2 & -2399 & 2 & 1 \\
\hline-2063 & 2 & 1 & -2235 & $(2,2,27)$ & $(2,2,9)$ & -2404 & 4 & 2 \\
\hline-2067 & $(2,2)$ & $(2,2)$ & -2239 & 2 & 1 & -2407 & 2 & 1 \\
\hline-2068 & 2 & 2 & -2243 & 1 & 1 & -2408 & $(2,3)$ & $(2,3)$ \\
\hline-2071 & 2 & 1 & -2244 & $(2,8,3)$ & $(2,4)$ & -2411 & 1 & 1 \\
\hline-2072 & 2 & 2 & -2247 & $(2,2)$ & 2 & -2415 & $(2,2,2,3)$ & $(2,2)$ \\
\hline-2083 & 1 & 1 & -2248 & 2 & 2 & -2419 & 4 & 4 \\
\hline-2084 & 4 & 2 & -2251 & 1 & 1 & -2423 & 2 & 1 \\
\hline-2087 & 2 & 1 & -2255 & $(2,2)$ & 2 & -2424 & $(2,3)$ & 2 \\
\hline-2091 & $(2,2,3)$ & $(2,2)$ & -2260 & 2 & 2 & -2427 & 2 & 2 \\
\hline-2095 & 2 & 1 & -2263 & $(2,2)$ & 2 & -2431 & $(2,2)$ & 2 \\
\hline-2099 & 1 & 1 & -2264 & 1 & 1 & -2435 & 2 & 2 \\
\hline-2103 & $(2,5)$ & 5 & -2267 & 1 & 1 & -2436 & $(2,4)$ & $(2,2)$ \\
\hline-2104 & 2 & 2 & -2271 & $(2,3,5)$ & 5 & -2440 & 2 & 2 \\
\hline-2111 & 2 & 1 & -2276 & 4 & 2 & -2443 & 2 & 2 \\
\hline-2119 & 2 & 1 & -2279 & 2 & 1 & -2447 & $(2,7)$ & 7 \\
\hline-2120 & 2 & 2 & -2280 & $(2,2,3)$ & $(2,2)$ & -2451 & $|(2,4,3,7)|$ & $(2,4,7)$ \\
\hline-2123 & 2 & 2 & -2283 & $(2,3)$ & $(2,3)$ & -2452 & 1 & 1 \\
\hline-2127 & $(2,3)$ & 1 & -2287 & 2 & 1 & -2455 & 2 & 1 \\
\hline-2131 & 1 & 1 & -2291 & 2 & 2 & -2456 & 1 & 1 \\
\hline-2132 & $(2,3)$ & $(2,3)$ & -2292 & 2 & 2 & -2459 & 1 & 1 \\
\hline-2135 & $(2,2)$ & 2 & -2296 & $(2,2)$ & $(2,2)$ & -2463 & 2 & 1 \\
\hline-2136 & $(2,3)$ & 2 & -2307 & $(4,3)$ & 4 & -2467 & 1 & 1 \\
\hline-2139 & $(2,2)$ & $(2,2)$ & -2308 & 16 & 8 & -2468 & $(4,3)$ & $(2,3)$ \\
\hline-2143 & 2 & 1 & -2311 & 2 & 1 & -2471 & $(2,2)$ & 2 \\
\hline-2147 & 2 & 2 & -2315 & 2 & 2 & -2472 & 2 & 2 \\
\hline-2148 & 4 & 2 & -2319 & 2 & 1 & -2479 & 2 & 1 \\
\hline-2152 & 1 & 1 & -2323 & 2 & 2 & -2483 & 2 & 2 \\
\hline-2155 & 2 & 2 & -2324 & 2 & 2 & -2487 & $(2,3)$ & 1 \\
\hline-2159 & $(2,2)$ & 2 & -2327 & 2 & 1 & -2488 & $(2,3)$ & $(2,3)$ \\
\hline-2163 & $(2,2,3,5)$ & $(2,2,5)$ & -2328 & 4 & 4 & -2491 & $(2,3)$ & $(2,3)$ \\
\hline-2164 & 1 & 1 & -2335 & 2 & 1 & -2495 & 2 & 1 \\
\hline-2167 & 2 & 1 & -2339 & 1 & 1 & -2503 & 2 & 1 \\
\hline-2168 & 2 & 2 & -2343 & $(2,2,3)$ & 2 & -2504 & 2 & 2 \\
\hline
\end{tabular}


Table 1. (Continued)

\begin{tabular}{|c|c|c|c|c|c|c|c|c|}
\hline$d$ & tame & wild & $d$ & tame & \begin{tabular}{|l|} 
wild \\
\end{tabular} & $d$ & tame & wild \\
\hline-2507 & 2 & 2 & \begin{tabular}{|l|}
-2679 \\
\end{tabular} & $(2,2)$ & 2 & \begin{tabular}{|l|}
-2839 \\
\end{tabular} & $(2,2)$ & 2 \\
\hline-2515 & 2 & 2 & $|-2680|$ & 2 & 2 & -2840 & 2 & 2 \\
\hline-2516 & 2 & 2 & -2683 & 1 & 1 & -2843 & 1 & 1 \\
\hline-2519 & 2 & 1 & $|-2687|$ & 2 & 1 & -2847 & $(2,2,3)$ & 2 \\
\hline-2531 & 1 & 1 & $\mid-2692$ & 8 & 4 & -2851 & 1 & 1 \\
\hline-2532 & $(4,3)$ & 2 & $\mid-2696$ & 8 & 8 & -2852 & $(2,4,3)$ & $(2,2,3)$ \\
\hline-2536 & 1 & 1 & -2699 & 1 & 1 & -2855 & 2 & 1 \\
\hline-2539 & 1 & 1 & -2703 & $(2,2,3)$ & 2 & -2856 & $(2,2,9)$ & $(2,2,3)$ \\
\hline-2543 & 2 & 1 & -2707 & 1 & 1 & $\mid-2859$ & $(2,5)$ & $(2,5)$ \\
\hline-2551 & 2 & 1 & $\mid-2708$ & 1 & 1 & -2863 & $(2,2)$ & 2 \\
\hline-2552 & 2 & 2 & -2711 & 2 & 1 & -2867 & $(2,5)$ & $(2,5)$ \\
\hline-2555 & $(2,2,3)$ & $(2,2,3)$ & -2712 & $(2,3)$ & 2 & -2868 & 2 & 2 \\
\hline-2559 & $(2,3)$ & 1 & -2715 & $(2,2)$ & $(2,2)$ & -2872 & 2 & 2 \\
\hline-2563 & 2 & 2 & -2719 & 2 & 1 & $\mid-2879$ & $(2,3)$ & 3 \\
\hline-2564 & 8 & 4 & -2723 & 2 & 2 & -2884 & $(2,8)$ & $(2,4)$ \\
\hline-2567 & $(2,2)$ & 2 & -2724 & 4 & 2 & -2887 & 2 & 1 \\
\hline-2568 & $(2,3)$ & 2 & -2728 & 2 & 2 & -2895 & $(2,2)$ & 2 \\
\hline-2571 & 2 & 2 & -2731 & 1 & 1 & $\mid-2899$ & 2 & 2 \\
\hline-2579 & 1 & 1 & -2735 & 2 & 1 & $\mid-2903$ & 2 & 1 \\
\hline-2580 & $(2,2)$ & $(2,2)$ & -2739 & $(2,8,3)$ & $(2,8)$ & -2911 & $(2,2)$ & 2 \\
\hline-2584 & 8 & 8 & -2740 & $(2,3)$ & $(2,3)$ & -2915 & $(2,4,3)$ & $(2,4,3)$ \\
\hline-2587 & 2 & 2 & -2743 & 2 & 1 & $\mid-2919$ & $(2,4,3)$ & 4 \\
\hline-2591 & 2 & 1 & -2747 & 2 & 2 & -2920 & 2 & 2 \\
\hline-2595 & $(2,2,3)$ & $(2,2)$ & -2751 & $(2,4)$ & 4 & -2923 & $(2,3,23)$ & $(2,3,23)$ \\
\hline-2596 & 4 & 2 & -2755 & $(2,2)$ & $(2,2)$ & -2927 & 2 & 1 \\
\hline-2599 & $(2,2)$ & 2 & -2756 & 4 & 2 & -2931 & 8 & 2 \\
\hline-2603 & 4 & 4 & -2759 & $(2,2,3)$ & $(2,3)$ & -2932 & 1 & 1 \\
\hline-2607 & $(2,2)$ & 2 & -2760 & $(2,2)$ & $(2,2)$ & -2935 & 2 & 1 \\
\hline-2611 & 2 & 2 & -2767 & $(2,5)$ & 5 & -2936 & 2 & 2 \\
\hline-2612 & 1 & 1 & -2771 & 2 & 2 & -2939 & 1 & 1 \\
\hline-2615 & 2 & 1 & -2776 & 11 & 11 & -2947 & 2 & 2 \\
\hline-2616 & 2 & 2 & -2779 & 0 & 0 & -2948 & 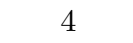 & 2 \\
\hline-2623 & 2 & 1 & -6787 & 2 & 2 & $\mid-2951$ & $(2,5)$ & 5 \\
\hline-2627 & $(2,3)$ & $(2,3)$ & -2788 & $(2,4)$ & $(2,2)$ & -2955 & $(2,2,3)$ & $(2,2)$ \\
\hline-2631 & $(2,3)$ & 1 & -2791 & $(2,3)$ & 3 & -2959 & 0 & 1 \\
\hline-2632 & $(2,2)$ & $(2,2)$ & -2792 & 5 & 5 & -2963 & 1 & 1 \\
\hline-2635 & $(2,2)$ & $(2,2)$ & -2795 & $(2,2)$ & $(2,2)$ & -2964 & $(2,2,3)$ & $(2,2)$ \\
\hline-2639 & $(2,2)$ & 2 & -2803 & 1 & 1 & -2967 & $(2,2)$ & 2 \\
\hline-2643 & 2 & 2 & -2804 & 1 & 1 & -2968 & 2 & 2 \\
\hline-2644 & 1 & 1 & -2807 & $(2,4)$ & 4 & -2971 & 5 & 5 \\
\hline-2647 & 2 & 1 & -2811 & $(32,3)$ & 32 & -2980 & 4 & 2 \\
\hline-2648 & 1 & 1 & -2815 & 2 & 1 & $\mid-2983$ & 2 & 1 \\
\hline-2651 & 2 & 2 & -2819 & 1 & 1 & -2984 & 1 & 1 \\
\hline-2659 & 1 & 1 & -2820 & $(2,4,3)$ & $(2,2)$ & -2987 & 2 & 2 \\
\hline-2660 & $(2,4)$ & $(2,2)$ & -2823 & 2 & 1 & $\mid-2991$ & $(2,3)$ & 1 \\
\hline-2663 & 2 & 1 & -2824 & $(8,3)$ & $(8,3)$ & -2995 & 2 & 2 \\
\hline-2667 & $(2,2,3)$ & $(2,2)$ & -2827 & $(4,5)$ & $(4,5)$ & -2996 & 2 & 2 \\
\hline-2671 & 2 & 1 & -2831 & 2 & 1 & $\mid-2999$ & 2 & 1 \\
\hline-2676 & $(2,3)$ & 2 & -2836 & 1 & 1 & -3003 & $(2,2,2)$ & $(2,2,2)$ \\
\hline
\end{tabular}


Table 1. (Continued)

\begin{tabular}{|c|c|c|}
\hline$d$ & tame & wild \\
\hline-3007 & $(2,4)$ & 4 \\
-3011 & 7 & 7 \\
-3012 & 4 & 2 \\
-3016 & 2 & 2 \\
-3019 & 7 & 7 \\
-3023 & 2 & 1 \\
-3027 & $(4,3)$ & 4 \\
-3028 & 1 & 1 \\
-3031 & $(2,2)$ & 2 \\
-3032 & 1 & 1 \\
-3035 & 2 & 2 \\
-3039 & 2 & 1 \\
-3043 & 4 & 4 \\
-3044 & 4 & 2 \\
-3047 & $(2,5)$ & 5 \\
-3048 & $(2,5,9)$ & $(2,5,9)$ \\
-3055 & $(2,2,5)$ & $(2,5)$ \\
-3059 & $(2,2)$ & $(2,2)$ \\
-3063 & $(2,3)$ & 1 \\
-3064 & $(2,3)$ & $(2,3)$ \\
-3067 & 1 & 1 \\
-3071 & 2 & 1 \\
-3076 & 8 & 4 \\
-3079 & 2 & 1 \\
-3080 & $(2,2)$ & $(2,2)$ \\
-3083 & 1 & 1 \\
-3091 & 2 & 2 \\
-3092 & 1 & 1 \\
-3095 & 2 & 1 \\
-3099 & $(4,3)$ & 4 \\
-3103 & 2 & 1 \\
-3107 & 2 & 2 \\
-3108 & $(2,4,3,13)$ & $(2,2,13)$ \\
-3111 & $(2,2)$ & 2 \\
-3112 & 1 & 1 \\
-3115 & $(2,2)$ & $(2,2)$ \\
-3119 & 2 & 1 \\
-3124 & 2 & 2 \\
-3127 & 2 & 1 \\
-3128 & $(2,2)$ & $(2,2)$ \\
-3131 & 2 & 2 \\
-3135 & $(2,2,2,3)$ & $(2,2)$ \\
-3139 & 2 & 2 \\
-3140 & 4 & 2 \\
-3143 & $(2,2)$ & 2 \\
-3144 & $(2,3)$ & 2 \\
-3147 & 2 & 2 \\
-3151 & $(2,2)$ & 2 \\
-3155 & 2 & 2 \\
-3156 & 2 & 2 \\
-3160 & 2 & 2 \\
\hline & & \\
\hline
\end{tabular}

\begin{tabular}{|c|c|c|}
\hline$d$ & tame & wild \\
\hline-3163 & 1 & 1 \\
-3167 & 2 & 1 \\
-3171 & $(2,2,3)$ & $(2,2)$ \\
-3172 & 4 & 2 \\
-3176 & 17 & 17 \\
-3183 & 2 & 1 \\
-3187 & 1 & 1 \\
-3188 & 1 & 1 \\
-3191 & 2 & 1 \\
-3192 & $(2,2)$ & $(2,2)$ \\
-3199 & $(2,2)$ & 2 \\
-3203 & 1 & 1 \\
-3207 & $(2,3)$ & 1 \\
-3208 & 4 & 4 \\
-3215 & $(2,23)$ & 23 \\
-3219 & $(2,2)$ & $(2,2)$ \\
-3220 & $(2,2)$ & $(2,2)$ \\
-3223 & 2 & 1 \\
-3224 & $(2,7)$ & $(2,7)$ \\
-3227 & 2 & 2 \\
-3235 & 2 & 2 \\
-3236 & 4 & 2 \\
-3239 & $(2,2)$ & 2 \\
-3243 & $(2,4,3)$ & $(2,4)$ \\
-3247 & $(2,16)$ & 16 \\
-3251 & 1 & 1 \\
-3252 & $(2,3)$ & 2 \\
-3255 & $(2,2,2)$ & $(2,2)$ \\
-3256 & 2 & 2 \\
-3259 & 1 & 1 \\
-3263 & 2 & 1 \\
-3268 & 4 & 2 \\
-3271 & $(2,3)$ & 3 \\
-3272 & 2 & 2 \\
-3279 & $(2,3)$ & 1 \\
-3284 & 1 & 1 \\
-3287 & 2 & 1 \\
-3288 & $(2,3)$ & 2 \\
-3291 & 2 & 2 \\
-3295 & 2 & 1 \\
-3299 & $(3,5)$ & $(3,5)$ \\
-3304 & 2 & 2 \\
-3307 & 1 & 1 \\
-3311 & $(2,2)$ & 2 \\
-3315 & $(2,2,2,3)$ & $(2,2,2)$ \\
-3316 & 1 & 1 \\
-3319 & 2 & 1 \\
-3320 & $(2,5)$ & $(2,5)$ \\
-3323 & 1 & 1 \\
-3327 & 2 & 1 \\
-3331 & 1 & 1 \\
\hline & &
\end{tabular}

\begin{tabular}{|c|c|c|}
\hline$d$ & tame & wild \\
\hline-3335 & $(2,2)$ & 2 \\
-3336 & 2 & 2 \\
-3343 & 2 & 1 \\
-3347 & 1 & 1 \\
-3351 & $(2,3)$ & 1 \\
-3352 & 1 & 1 \\
-3355 & $(2,2,13)$ & $(2,2,13)$ \\
-3359 & $(2,3)$ & 3 \\
-3363 & $(2,4)$ & $(2,4)$ \\
-3367 & $(2,2)$ & 2 \\
-3368 & 1 & 1 \\
-3371 & 1 & 1 \\
-3379 & $(2,37)$ & $(2,37)$ \\
-3383 & $(2,2)$ & 2 \\
-3387 & $(4,3,3)$ & $(4,3)$ \\
-3391 & 2 & 1 \\
-3395 & $(2,2)$ & $(2,2)$ \\
-3396 & $(4,3)$ & 2 \\
-3399 & $(2,4)$ & 4 \\
-3403 & 8 & 8 \\
-3407 & 2 & 1 \\
-3412 & 1 & 1 \\
-3415 & 2 & 1 \\
-3416 & $(2,11)$ & $(2,11)$ \\
-3419 & 2 & 2 \\
-3423 & $(2,2,3,11)$ & $(2,11)$ \\
-3427 & 2 & 2 \\
-3428 & 4 & 2 \\
-3431 & $(2,2)$ & 2 \\
-3432 & $(2,2,3)$ & $(2,2)$ \\
-3435 & $(2,2)$ & $(2,2)$ \\
-3439 & 2 & 1 \\
-3443 & 4 & 4 \\
-3444 & $(2,2)$ & $(2,2)$ \\
-3448 & 2 & 2 \\
-3451 & $(2,2,3)$ & $(2,2,3)$ \\
-3455 & 2 & 1 \\
-3459 & $(4,9)$ & $(4,3)$ \\
-3460 & 4 & 2 \\
-3463 & 2 & 1 \\
-3464 & 4 & 4 \\
-3467 & 1 & 1 \\
-3471 & $(2,2)$ & 2 \\
-3476 & 2 & 2 \\
-3480 & $(2,4)$ & $(2,4)$ \\
-3487 & 2 & 1 \\
-3491 & 1 & 1 \\
-3495 & $(2,2,3)$ & 2 \\
-3496 & 2 & 2 \\
-3499 & 1 & 1 \\
-3503 & $(2,2)$ & 2 \\
\hline & &
\end{tabular}


Table 1. (Continued)

\begin{tabular}{|c|c|c|c|c|c|c|c|c|}
\hline$d$ & tame & wild & $d$ & tame & wild & $d$ & tame & wild \\
\hline-3507 & $(2,2)$ & $(2,2)$ & \begin{tabular}{|l|}
-3659 \\
\end{tabular} & 1 & 1 & \begin{tabular}{|l|}
-3832 \\
\end{tabular} & 2 & 2 \\
\hline-3508 & 1 & 1 & -3667 & 2 & 2 & -3835 & $(2,2,3,23)$ & $(2,2,3,23)$ \\
\hline-3511 & 2 & 1 & -3668 & 2 & 2 & -3839 & 2 & 1 \\
\hline-3512 & 2 & 2 & $\mid-3671$ & $(2,3)$ & 3 & $\mid-3847$ & 2 & 1 \\
\hline-3515 & $(2,2)$ & $(2,2)$ & -3679 & 2 & 1 & $\mid-3848$ & 2 & 2 \\
\hline-3523 & 2 & 2 & -3683 & 2 & 2 & $\mid-3851$ & 1 & 1 \\
\hline$-3524 \mid$ & 16 & 8 & -3684 & $(4,3)$ & 2 & -3855 & $(2,2,3)$ & 2 \\
\hline-3527 & 2 & 1 & -3687 & $(2,3)$ & 3 & -3859 & 2 & 2 \\
\hline-3531 & $(2,4,3)$ & $(2,4)$ & -3688 & 1 & 1 & -3860 & 2 & 2 \\
\hline-3535 & $(2,2)$ & 2 & $\mid-3691$ & 1 & 1 & $\mid-3863$ & 2 & 1 \\
\hline-3539 & 1 & 1 & -3695 & $(2,3,5)$ & $(3,5)$ & -3864 & $(2,2,3)$ & $(2,2)$ \\
\hline-3540 & $(2,2,27)$ & $(2,2,9)$ & -3704 & 2 & 2 & $\mid-3867$ & 2 & 2 \\
\hline-3543 & 2 & 1 & -3707 & 2 & 2 & $\mid-3876$ & $(2,4)$ & $(2,2)$ \\
\hline-3544 & 1 & 1 & -3711 & $(2,3)$ & 1 & $\mid-3880$ & 2 & 2 \\
\hline-3547 & 3 & 3 & -3715 & 2 & 2 & -3883 & 8 & 8 \\
\hline$-3551 \mid$ & 2 & 1 & -3716 & 8 & 4 & $\mid-3891$ & $(4,3)$ & 4 \\
\hline-3556 & $(2,4)$ & $(2,2)$ & -3719 & 2 & 1 & $\mid-3892$ & 2 & 2 \\
\hline-3559 & 2 & 1 & -3720 & $(2,2,3)$ & $(2,2)$ & $\mid-3895$ & $(2,2)$ & 2 \\
\hline$-3560 \mid$ & 4 & 4 & -3723 & $(2,2,5)$ & $(2,2,5)$ & $\mid-3896$ & $(2,3)$ & $(2,3)$ \\
\hline-3563 & 2 & 2 & -3727 & 2 & 1 & $\mid-3899$ & $(2,3)$ & $(2,3)$ \\
\hline-3567 & $(2,2,3)$ & 2 & -3731 & $(2,2)$ & $(2,2)$ & $\mid-3903$ & 2 & 1 \\
\hline$-3571 \mid$ & 1 & 1 & -3732 & 2 & 2 & $\mid-3907$ & 1 & 1 \\
\hline-3572 & 2 & 2 & -3736 & 1 & 1 & $\mid-3908$ & 8 & 4 \\
\hline-3576 & $(2,3)$ & 2 & -3739 & 1 & 1 & $\mid-3911$ & 2 & 1 \\
\hline-3579 & 2 & 2 & -3743 & 2 & 1 & $\mid-3912$ & $(2,3)$ & $(2,3)$ \\
\hline-3583 & 2 & 1 & -3747 & $(4,3)$ & 4 & $\mid-3919$ & $(2,3)$ & 3 \\
\hline-3587 & 2 & 2 & -7748 & 4 & 2 & $\mid-3923$ & 1 & 1 \\
\hline-3588 & $(2,4)$ & $(2,2)$ & -3752 & 2 & 2 & $\mid-3927$ & $(2,2,2,3)$ & $(2,2)$ \\
\hline-3592 & 4 & 4 & -3755 & 2 & 2 & $\mid-3928$ & 1 & 1 \\
\hline$-3595 \mid$ & 2 & ه & -379 & $(2,2)$ & 2 & $\mid-3931$ & 1 & 1 \\
\hline-3599 & 2 & 1 & -3763 & $(2,3)$ & $(2,3)$ & $\mid-3935$ & 2 & 1 \\
\hline-3603 & $(4,3)$ & 4 & -3764 & 1 & 1 & -3939 & $(2,2)$ & $(2,2)$ \\
\hline-3604 & $(4,3)$ & $(4,3)$ & $\mid-3767$ & 2 & 1 & $\mid-3940$ & 4 & 2 \\
\hline-3607 & $(2,17)$ & 17 & -3768 & 2 & 2 & $\mid-3943$ & $(2,3)$ & 3 \\
\hline-3608 & 2 & 2 & -3779 & 1 & 1 & $\mid-3944$ & 2 & 2 \\
\hline-3611 & 2 & 2 & -3783 & $(2,2,9)$ & $(2,3)$ & $\mid-3947$ & 1 & 1 \\
\hline$-3615 \mid$ & $(2,4)$ & 4 & -3784 & 2 & 2 & -3955 & $(2,2)$ & $(2,2)$ \\
\hline-3619 & $(2,2)$ & $(2,2)$ & $\mid-3787$ & 2 & 2 & $\mid-3956$ & $(2,5)$ & $(2,5)$ \\
\hline-3620 & 4 & 2 & -3791 & $(2,4)$ & 4 & $\mid-3959$ & $(2,23)$ & 23 \\
\hline-3623 & 2 & 1 & -3795 & $(2,2,2)$ & $(2,2,2)$ & -3963 & $(8,3)$ & 8 \\
\hline-3624 & $(2,125)$ & $(2,125)$ & -3796 & 2 & 2 & $\mid-3967$ & 2 & 1 \\
\hline-3631 & 2 & 1 & $\mid-3799$ & 2 & 1 & $\mid-3972$ & $(4,3)$ & 2 \\
\hline-3635 & 2 & 2 & -3803 & 1 & 1 & $\mid-3976$ & $(2,4)$ & $(2,4)$ \\
\hline-3639 & $(2,3)$ & 1 & -3811 & 2 & 2 & -3979 & 2 & 2 \\
\hline-3640 & $(2,4)$ & $(2,4)$ & -3812 & 4 & 2 & $\mid-3983$ & $(2,2)$ & 2 \\
\hline-3643 & 3 & 3 & -3815 & $(2,2)$ & 2 & $\mid-3988$ & 1 & 1 \\
\hline-3647 & $(2,2,3)$ & $(2,3)$ & -3819 & $(2,4,3)$ & $(2,4)$ & $\mid-3991$ & 2 & 1 \\
\hline-3651 & 2 & 2 & -3823 & 2 & 1 & $\mid-3992$ & 1 & 1 \\
\hline-3652 & 4 & 2 & -3827 & 2 & 2 & $\mid-3995$ & $(2,2)$ & $(2,2)$ \\
\hline-3655 & $(2,2)$ & 2 & -3828 & $(2,2,3)$ & $(2,2)$ & $\mid-3999$ & $(2,4,9)$ & $(4,3)$ \\
\hline-3656 & 2 & 2 & -3831 & 2 & 1 & -4003 & 1 & 1 \\
\hline
\end{tabular}


Table 1. (Continued)

\begin{tabular}{|c|c|c|c|c|c|c|c|c|}
\hline$d$ & tame & wild & $d$ & tame & wild & $d$ & tame & wild \\
\hline-4004 & $(2,4)$ & $(2,2)$ & -4179 & $(2,2,3)$ & $(2,2)$ & \begin{tabular}{|l|}
-4344 \\
\end{tabular} & 2 & 2 \\
\hline-4007 & 2 & 1 & -4180 & $(2,4)$ & $(2,4)$ & -4351 & 2 & 1 \\
\hline-4008 & $(2,3)$ & 2 & -4183 & $(2,4)$ & 4 & -4355 & $(2,2)$ & $(2,2)$ \\
\hline-4011 & $(2,2)$ & $(2,2)$ & -4184 & 3 & 3 & -4359 & $(2,3)$ & 1 \\
\hline-4015 & $(2,2)$ & 2 & -4187 & 2 & 2 & -4360 & 2 & 2 \\
\hline-4019 & 5 & 5 & -4191 & $(2,2)$ & 2 & -4363 & 3 & 3 \\
\hline-4020 & $(2,2)$ & $(2,2)$ & -4195 & 2 & 2 & -4367 & 2 & 1 \\
\hline-4024 & 2 & 2 & -4196 & 4 & 2 & -4371 & $(2,2,49)$ & $(2,2,49)$ \\
\hline-4027 & 3 & 3 & -4199 & $(2,4)$ & 4 & -4372 & 1 & 1 \\
\hline-4031 & 2 & 1 & -4207 & $(2,2)$ & 2 & -4376 & 1 & 1 \\
\hline-4035 & $(2,2,3)$ & $(2,2)$ & -4211 & 1 & 1 & -4379 & 2 & 2 \\
\hline-4036 & 8 & 4 & -4215 & $(2,2,3)$ & 2 & -4387 & 8 & 8 \\
\hline-4039 & $(2,2)$ & 2 & -4216 & $(2,2)$ & $(2,2)$ & -4388 & 4 & 2 \\
\hline-4040 & 2 & 2 & -4219 & 3 & 3 & -4391 & 2 & 1 \\
\hline-4043 & 2 & 2 & -4223 & $(2,4)$ & 4 & -4395 & $(2,2,3)$ & $(2,2)$ \\
\hline-4047 & $(2,2)$ & 2 & -4227 & 2 & 2 & -4399 & 2 & 1 \\
\hline-4051 & 1 & 1 & -4228 & $(2,8)$ & $(2,4)$ & -4403 & $(2,2)$ & $(2,2)$ \\
\hline-4052 & 1 & 1 & -4231 & 2 & 1 & -4404 & $(2,3)$ & 2 \\
\hline-4055 & 2 & 1 & -4243 & 5 & 5 & -4407 & $(2,2)$ & 2 \\
\hline-4063 & $(2,4)$ & 4 & -4244 & 5 & 5 & -4408 & 2 & 2 \\
\hline-4071 & $(2,2,3)$ & 2 & -4247 & $(2,2)$ & 2 & -4411 & 4 & 4 \\
\hline-4072 & 3 & 3 & -4251 & $(2,4,3,7)$ & $(2,4,7)$ & -4415 & $(2,3,7)$ & $(3,7)$ \\
\hline-4079 & 2 & 1 & -4255 & $(2,2,3)$ & $(2,3)$ & -4420 & $(2,4)$ & $(2,2)$ \\
\hline-4083 & 2 & 2 & -4259 & 1 & 1 & -4423 & $(2,3)$ & 3 \\
\hline-4084 & 1 & 1 & -4260 & $(2,4,3)$ & $(2,2)$ & -4424 & $(2,2,5)$ & $(2,2,5)$ \\
\hline-4087 & 2 & 1 & -4264 & 2 & 2 & -4427 & 4 & 4 \\
\hline-4088 & $(2,2)$ & $(2,2)$ & -4267 & 4 & 4 & -4431 & $(2,2,3)$ & 2 \\
\hline-4091 & 1 & 1 & -4271 & 2 & 1 & -4435 & 2 & 2 \\
\hline-4099 & 1 & 1 & -4276 & 1 & 1 & -4436 & 1 & 1 \\
\hline-4103 & $(2,3)$ & 3 & -4279 & 2 & 1 & -4439 & $(2,2)$ & 2 \\
\hline-4111 & $(2,3)$ & 3 & -4280 & 2 & 2 & -4440 & $(2,2,3)$ & $(2,2)$ \\
\hline-4115 & 2 & 2 & -4283 & 3 & 3 & -4443 & 2 & 2 \\
\hline-4119 & $(2,3)$ & 3 & -4287 & $(2,3)$ & 1 & -4447 & 2 & 1 \\
\hline-4120 & 2 & 2 & -4291 & 2 & 2 & -4451 & 1 & 1 \\
\hline-4123 & $(2,2)$ & $(2,2)$ & -4292 & 4 & 2 & -4452 & $(2,4)$ & $(2,2)$ \\
\hline-4127 & 2 & 1 & -4295 & 2 & 1 & -4456 & 1 & 1 \\
\hline-4132 & 4 & 2 & -4296 & $(2,3)$ & 2 & -4463 & 2 & 1 \\
\hline-4135 & 2 & 1 & -4299 & 2 & 2 & -4467 & $(4,3)$ & 4 \\
\hline-4136 & 2 & 2 & -4303 & 2 & 1 & -4468 & 1 & 1 \\
\hline-4139 & 7 & 7 & -4307 & $(2,5)$ & $(2,5)$ & -4471 & $(2,2)$ & 2 \\
\hline-4143 & $(2,3)$ & 1 & -4308 & $(2,3)$ & $(2,3)$ & -4472 & 2 & 2 \\
\hline-4147 & $(2,2,3)$ & $(2,2,3)$ & -4315 & 2 & 2 & -4479 & 2 & 1 \\
\hline-4148 & $(2,29)$ & $(2,29)$ & -4319 & $(2,4,5)$ & $(4,5)$ & -4483 & 1 & 1 \\
\hline-4151 & $(2,2,5)$ & $(2,5)$ & -4323 & $(2,4,3)$ & $(2,4)$ & -4484 & 4 & 2 \\
\hline-4152 & $(2,3)$ & 2 & -4324 & $(2,4)$ & $(2,2)$ & -4487 & $(2,4)$ & 4 \\
\hline-4155 & $(2,2)$ & $(2,2)$ & -4327 & 2 & 1 & -4488 & $(2,4)$ & $(2,4)$ \\
\hline-4159 & $(2,5)$ & 5 & -4328 & 1 & 1 & -4495 & $(2,2)$ & 2 \\
\hline-4163 & 2 & 2 & -4331 & $(2,7)$ & $(2,7)$ & -4499 & 2 & 2 \\
\hline-4164 & 4 & 2 & -4339 & 1 & 1 & -4503 & $(2,2,3)$ & 2 \\
\hline-4168 & 2 & 2 & -4340 & $(2,2)$ & $(2,2)$ & -4504 & 1 & 1 \\
\hline-4171 & 4 & 4 & -4343 & 2 & 1 & -4507 & 1 & 1 \\
\hline
\end{tabular}


Table 1. (Continued)

\begin{tabular}{|c|c|c|c|c|c|c|c|c|}
\hline$d$ & tame & wild & $d$ & tame & wild & $d$ & tame & wild \\
\hline-4511 & 2 & 1 & -4676 & $(2,8)$ & $(2,4)$ & -4836 & $(2,4,3)$ & $(2,2)$ \\
\hline-4515 & $(2,2,2)$ & $(2,2,2)$ & -4679 & 2 & 1 & -4839 & 2 & 1 \\
\hline-4516 & 4 & 2 & -4683 & $(2,2,3,37)$ & $(2,2,37)$ & -4843 & 2 & 2 \\
\hline-4519 & 2 & 1 & -4687 & 2 & 1 & -4847 & 2 & 1 \\
\hline-4520 & 2 & 2 & -4691 & 1 & 1 & -4852 & 1 & 1 \\
\hline-4523 & 1 & 1 & -4692 & $(2,2,3)$ & $(2,2)$ & -4855 & 2 & 1 \\
\hline-4531 & 2 & 2 & -4695 & $(2,2)$ & 2 & -4856 & 2 & 2 \\
\hline-4532 & 2 & 2 & -4696 & 1 & 1 & -4859 & 2 & 2 \\
\hline-4535 & 2 & 1 & -4699 & 2 & 2 & -4863 & $(2,3)$ & 1 \\
\hline-4539 & $(2,2,3)$ & $(2,2)$ & -4703 & 2 & 1 & -4867 & 2 & 2 \\
\hline-4543 & $(2,2)$ & 2 & -4708 & 4 & 2 & -4868 & 64 & 32 \\
\hline-4547 & 233 & 233 & -4711 & $(2,2)$ & 2 & -4871 & 2 & 1 \\
\hline-4548 & $(4,3)$ & 2 & -4712 & 2 & 2 & -4872 & $(2,2,3)$ & $(2,2)$ \\
\hline-4551 & $(2,2)$ & 2 & -4715 & $(2,4)$ & $(2,4)$ & -4879 & $(2,2,2)$ & $(2,2)$ \\
\hline-4552 & 2 & 2 & -4723 & 1 & 1 & -4883 & 2 & 2 \\
\hline-4555 & 2 & 2 & -4724 & 1 & 1 & -4884 & $(2,16)$ & $(2,16)$ \\
\hline-4559 & $(2,2)$ & 2 & -4727 & 2 & 1 & -4888 & $(2,5,7)$ & $(2,5,7)$ \\
\hline-4564 & 2 & 2 & -4728 & $(2,3)$ & 2 & -4891 & 4 & 4 \\
\hline-4567 & 2 & 1 & -4731 & $(2,2)$ & $(2,2)$ & -4895 & $(2,4)$ & 4 \\
\hline-4568 & 1 & 1 & -4735 & 2 & 1 & -4899 & $(2,8,3)$ & $(2,8)$ \\
\hline-4571 & 2 & 2 & -4739 & 2 & 2 & -4903 & $(2,5)$ & 5 \\
\hline-4579 & $(2,5)$ & $(2,5)$ & -4740 & $(2,4)$ & $(2,2)$ & -4904 & 1 & 1 \\
\hline-4580 & 4 & 2 & -4744 & 8 & 8 & -4907 & 2 & 2 \\
\hline-4583 & $(2,5)$ & 5 & -4747 & 2 & 2 & -4911 & 2 & 1 \\
\hline-4584 & $(2,3)$ & 2 & -4751 & 2 & 1 & -4915 & 2 & 2 \\
\hline-4587 & $(2,2)$ & $(2,2)$ & -4755 & $(2,2,3)$ & $(2,2)$ & -4916 & 1 & 1 \\
\hline-4591 & 2 & 1 & -4756 & 2 & 2 & -4919 & 2 & 1 \\
\hline-4595 & $(2,3)$ & $(2,3)$ & -4759 & 2 & 1 & -4920 & $(2,2)$ & $(2,2)$ \\
\hline-4596 & 2 & 2 & -4760 & $(2,2)$ & $(2,2)$ & -4927 & 2 & 1 \\
\hline-4603 & 1 & 1 & -4763 & 4 & 4 & -4931 & 1 & 1 \\
\hline-4607 & $(2,4)$ & 4 & -4767 & $(2,4)$ & 4 & -4935 & $(2,2,2,3)$ & $(2,2)$ \\
\hline-4611 & $(2,2,3)$ & $(2,2)$ & -4771 & $(2,7)$ & $(2,7)$ & -4936 & $(2,5)$ & $(2,5)$ \\
\hline-4612 & 8 & 4 & -4772 & $(4,3,5)$ & $(2,3,5)$ & -4939 & 4 & 4 \\
\hline-4615 & $(2,2)$ & 2 & -4776 & 2 & 2 & -4943 & 2 & 1 \\
\hline-4616 & 8 & 8 & -4783 & $(2,5)$ & 5 & -4947 & $(2,2)$ & $(2,2)$ \\
\hline-4619 & 2 & 2 & -4787 & 1 & 1 & -4948 & 17 & 17 \\
\hline-4623 & $(2,2)$ & 2 & -4791 & $(2,3)$ & 1 & -4951 & 2 & 1 \\
\hline-4627 & 2 & 2 & -4792 & $(2,9)$ & $(2,9)$ & -4952 & 1 & 1 \\
\hline-4628 & 2 & 2 & -4795 & $(2,2,3,7)$ & $(2,2,3,7)$ & -4955 & 2 & 2 \\
\hline-4631 & 2 & 1 & -4799 & $(2,3)$ & 3 & -4963 & 2 & 2 \\
\hline-4632 & $(4,7)$ & $(4,7)$ & -4803 & 2 & 2 & -4964 & $(2,4)$ & $(2,2)$ \\
\hline-4639 & 2 & 1 & -4804 & 8 & 4 & -4967 & 2 & 1 \\
\hline-4643 & 1 & 1 & -4807 & $(2,4)$ & 4 & -4971 & $(16,3)$ & 16 \\
\hline-4647 & $(2,3)$ & 1 & -4808 & $(2,3)$ & $(2,3)$ & -4979 & 2 & 2 \\
\hline-4648 & 2 & 2 & -4811 & 2 & 2 & -4980 & $(2,2,3)$ & $(2,2)$ \\
\hline-4651 & 1 & 1 & -4819 & $(2,3)$ & $(2,3)$ & -4983 & $(2,2)$ & 2 \\
\hline-4659 & 2 & 2 & $\begin{array}{lll}-48 & 20\end{array}$ & 4 & 4 & -4984 & $(2,2)$ & $(2,2)$ \\
\hline-4660 & 2 & 2 & -4823 & $(2,2)$ & 2 & -4987 & 1 & 1 \\
\hline-4663 & 2 & 1 & -4827 & $(4,9)$ & $(4,3)$ & -4991 & $(2,2,2)$ & $(2,2)$ \\
\hline-4664 & 2 & 2 & -4831 & 2 & 1 & -4996 & 32 & 16 \\
\hline-4667 & 2 & 2 & -4835 & $(2,3)$ & $(2,3)$ & -4999 & 2 & 1 \\
\hline
\end{tabular}




\section{REFERENCES}

[BBCO] C. Bernardi. D. Batut, H. Cohen and M. Olivier, GP-PARI, a computer package.

[Bl] S. Bloch, Applications of the dilogarithm function in algebraic K-theory and algebraic geometry, Proc. Int. Symp. Alg. Geom., Kyoto, Kinokuniya, 1977, pp. 103-114. MR 82f: 14009

[Bo1] A. Borel, Cohomologie de $S L_{n}$ et valeurs de fonctions zêta aux points entiers, Ann. Sc. Norm. Sup. Pisa (4) 4, no. 4 (1977), 613-636; errata 7, no. 2 (1980), 373. MR 58:22016; MR 81k:12012

[Bo2] A. Borel, Values of zeta-functions at integers, cohomology and polylogarithms, Current Trends in Mathematics and Physics, 1-44, Narosa, New Delhi, 1995. MR 97a:19005

[B-82] J. Browkin, The functor $K_{2}$ for the ring of integers of a number field, Universal Algebra and Applications, (Warsaw, 1978), Banach Center Publications, vol. 9, PWN, Warsaw, 1982, pp. 187-195. MR 85f:11084

[B-92] J. Browkin, On the p-rank of the tame kernel of algebraic number fields, Journ. Reine Angew. Math., 432 (1992), 135-149. MR 93j:11077

[B-S] J. Browkin and A. Schinzel, On Sylow 2-subgroups of $\mathrm{K}_{2} \mathrm{O}_{F}$ for quadratic number fields $F$, Journ. Reine Angew. Math., 331 (1982), 104-113. MR 83g:12011

[C-H] P. E. Conner and J. Hurrelbrink, Class number parity, Series in Pure Math. 8, World Scientific Publ, Singapore, 1988. MR 90f: 11092

[Ga] H. Gangl, Werte von Dedekindschen Zetafunktionen, Dilogarithmuswerte und Pflasterungen des hyperbolischen Raumes, Diplomarbeit Bonn, 1989.

[Gr] D. Grayson, Dilogarithm computations for $K_{3}$ in: Algebraic K-theory, Evanston 1980 (Proc. Conf., Northwestern Univ., Evanston, Ill., 1980), Lecture Notes in Math. 854 (1981), 168-178. MR 82i:12012

[KNF] M. Kolster, T. Nguyen Quang Do, V. Fleckinger, Twisted S-units, $p$-adic class number formulas, and the Lichtenbaum conjectures, Duke Math. J., 84 (1996), 679-717; errata 90 (1997), 641-643. MR 97g:11136; CMP 98:04

[Li] S. Lichtenbaum, Values of zeta-functions, étale cohomology, and algebraic K-theory, Lecture Notes in Math. 342 (1973), 489-501 Springer, Berlin. MR 53:10765

$[\mathrm{M}-\mathrm{W}]$ B. Mazur, A. Wiles, Class fields of abelian extensions of $\mathbb{Q}$, Invent. Math. 76, no. 2 (1984), 179-330. MR 85m:11069

[Q1] Qin Hourong, The 2-Sylow subgroups of the tame kernel of imaginary quadratic fields, Acta Arith., 69 (1995), 153-169. MR 96a:11132

[Q2] Qin Hourong, Computation of $K_{2} Z[\sqrt{-6}]$, Journ. Pure Appl. Algebra 96 (1994), 133-146. MR 95i: 11135

[Q3] Qin Hourong, Computation of $K_{2} Z\left[\frac{1+\sqrt{-35}}{2}\right]$, Chin. Ann. of Math., 17B, 1 (1996), 63-72. MR 97a:19004

[Sk] M. Skatba, Generalization of Thue's theorem and computation of the group $\mathrm{K}_{2} \mathrm{O}_{F}$, J. Number Theory 46 (1994), 303-322. MR 95d:19001

[Su] A.A. Suslin, Algebraic K-theory of fields, in: Proceedings of the International Congress of Mathematicians, Berkeley, CA, 1986, Vol.I, AMS, Providence, RI, 1987, pp. 222-244. MR 89k: 12010

[Ta] J. Tate, Appendix to "The Milnor ring of a global field" by H. Bass and J. Tate in: Algebraic K-theory, II: "Classical" algebraic K-theory and connections with arithmetic (Proc. Conf., Seattle Res. Center, Battelle Memorial Inst., 1971), Lecture Notes in Math. 342 (1973), 429-446. MR 56:449

Jerzy Browkin, Institute of Mathematics, University of Warsaw, ul. Banacha 2, PL02-097 Warszawa, Poland

E-mail address: bro@mimuw.edu.pl

Herbert Gangl, Institute for Experimental Mathematics, Ellernstr. 29, D-45326 Essen, Germany

E-mail address: herbert@mpim-bonn.mpg.de 